\title{
Treat all COVID 19-positive patients, but do not forget those negative with chronic diseases
}

\author{
Viganò Mauro ${ }^{1} \cdot$ Mantovani Lorenzo $0^{2,3} \cdot$ Cozzolino Paolo $^{3} \cdot$ Harari Sergio ${ }^{4}(\mathbb{D}$
}

Received: 28 April 2020 / Accepted: 1 June 2020 / Published online: 9 June 2020

(c) Società Italiana di Medicina Interna (SIMI) 2020

\begin{abstract}
The outbreak of coronavirus disease 2019 (COVID-19) has distressed our working practice. Infectious disease specialists, pneumologists and intensivists were not enough to face the enormous amount of patients that needed hospital care; therefore, many doctors have been recruited from other medical specialties trying to take care of as many patients as possible. The 'call to duty' of such doctors for urgent COVID-19 cases, however, diverted the attention from the care of patients with chronic conditions, which might have been neglected or undervalued. In this extremely difficult time, the standard of care of chronic patients has been reduced and this might have determined an increased rate of complications secondary to undermanagement. Thousands of patients with acute and chronic non-COVID-19 conditions have not accessed specialist care in the last weeks in Italy. Moreover, even those patients who have had scheduled an outpatient visit did not attend it for fear of leaving their home or due to the inability to go. During the pandemic, there was a drastic reduction in the number of hospital admissions for any medical conditions different from COVID-19. Self-presentation to the emergency department (ED) has been discouraged and the patients' own fear of being infected by going to the hospital led to also a significant decrease in ED access. During the lockdown, in San Giuseppe Hospital MultiMedica IRCCS, Milan, the ED admissions dropped from the mean of 2361/ month in December 2019-February 2020 to 1102 (- 53\%) and 861 (-63\%) in March and April 2020, respectively. For all the above-mentioned reasons, it is possible that some clinical conditions will further progress with a significant increase in morbidity and mortality. To prevent this, it is essential that patients with chronic conditions should be at least monitored and managed with telephone or online health consultation, identifying those who need urgent access to care, prioritizing outpatient visits based on disease severity. Patients with mild conditions could be managed outside the hospital by implementing telemedicine and creating networks of general practitioners who can consult with in-hospital specialists.
\end{abstract}

Keywords Chronic disease $\cdot$ COVID-19 $\cdot$ SARS-CoV-2 $\cdot$ Emergency department

Newly identified SARS CoV-2 may cause mild and selflimiting illness with upper respiratory tract symptoms,

Harari Sergio

sergio@sergioharari.it

1 Division of Hepatology, San Giuseppe Hospital MultiMedica IRCCS, Università Degli Studi Di Milano, Milan, Italy

2 Center for Public Health Research, University of Milano-Bicocca, Milan, Italy

3 Value-Based Healthcare Unit, IRCCS MultiMedica, Sesto San Giovanni, Italy

4 Dept of Medical Sciences, Dept of Clinical Sciences and Community Health, San Giuseppe Hospital MultiMedica IRCCS, Università Degli Studi Di Milano, Via San Vittore 12, 20123 Milan, Italy non-life-threatening pneumonia and severe pneumonia with acute respiratory distress syndrome (ARDS) that begin with mild symptoms and then progress rapidly to respiratory failure requiring intensive care management [1-3]. This latter occurrence affects above all older people or those with comorbidities, i.e., diabetes, arterial hypertension, pulmonary disease, and other chronic conditions [1]. The current outbreak of coronavirus disease 2019 (COVID-19) has been classified by the World Health Organization as a global pandemic. Up to April 24, 2020 in Italy, the most affected European country, there had been 192.994 confirmed SARSCoV-2 infections and 25.969 deaths [4].

The COVID-19 pandemic has distressed our working practice, as health-care providers, as the number of infected patients grew in our hospitals. Infectious disease specialists, pneumologists and intensivists were not enough to 
face the enormous amount of patients that needed hospital care, therefore many doctors have been recruited from other medical specialities, often completely different, trying to take care of as many patients as possible. Endocrinologists, cardiologists, gastroenterologists, haematologists had to refresh their knowledge on interpreting arterial blood gas analysis, managing non-invasive ventilators and prescribing antiretroviral drugs in respiratory care units (ICU). The 'call to duty' of doctors for urgent COVID-19 cases, however, diverted the attention from the care of patients with chronic condition which might have been neglected or undervalued. In this extremely difficult time, the standard of care of chronic patients has been reduced and this might have determined an increased rate of complications secondary to undermanagement.

Thousands of patients with acute and chronic nonCOVID-19 conditions have not accessed specialist care in the last weeks in Italy. Moreover, even those patients who have had scheduled an outpatient visit did not attend it for fear of leaving their home, the inability to go, due to problems of public transport, or the lack of someone who can bring them to the clinic.
Self-presentation to the emergency department (ED) has been discouraged and the patients' own fear of being infected by going to hospital led to a further reduction in ED access [5]. By analysing the ED admissions during the COVID-19 lockdown in San Giuseppe Hospital MultiMedica IRCCS, Milan, we compare the data from March/ April 2020 to December 2019-February 2020 (Table 1). The number of patients admitted to the ED dropped from the mean of 2361 patients/month to $1102(-53 \%)$ and 861 (-63\%) in March and April 2020, respectively. The ED admissions by medical specialities showed a significant drop in traumatology, dermatology, gastroenterology/ hepatology and cardiology, with an increase of accesses for respiratory diseases (from a mean of $12 \%$ in previous months to 36\% and 29\% in March and April 2020, respectively), with a significant increase in some presentation symptoms at triage such as fever, cough and dyspnoea that increased from a mean of 3\%/month to $6 \%, 6 \%$ and $12 \%$, respectively. At the same time, red codes at triage admission doubled (3.9\% in March and 2.7\% in April 2020) compared to the $1.5 \%$ of the prior 3 months, with a concomitant significant reduction of white codes.
Table 1. Variations in the emergency department (ED) admittances from March/April 2020 compared to December 2019-February 2020

\begin{tabular}{|c|c|c|c|c|c|}
\hline & December 2019 & January 2020 & February 2020 & March 2020 & April 2020 \\
\hline \multicolumn{6}{|l|}{ ED access, no. (\%) } \\
\hline Cardiology & $88(4)$ & $100(4)$ & $97(4.2)$ & $28(2.6)$ & $22(2.6)$ \\
\hline Dermatology & $114(5)$ & $148(6)$ & $150(6.4)$ & $36(3.3)$ & $5(0.6)$ \\
\hline Gastroenterology/hepatology & $298(13)$ & $300(12)$ & $314(13.5)$ & $124(11.2)$ & $81(9.4)$ \\
\hline Gynaecology/obstetrics & $306(13.5)$ & $293(11.8)$ & $261(11)$ & $128(11.6)$ & $151(17.5)$ \\
\hline Musculoskeletal & $325(14.4)$ & $256(10.3)$ & $114(5)$ & $24(2.2)$ & $23(2.7)$ \\
\hline Neurology & $283(12)$ & $215(8.7)$ & $227(9.8)$ & $98(8.9)$ & $71(8.2)$ \\
\hline Other & $308(13.5)$ & $461(18.5)$ & $553(24)$ & $184(16.7)$ & $197(22.9)$ \\
\hline Respiratory & $196(8.6)$ & $297(12)$ & $321(14)$ & $397(36)$ & 249 (29) \\
\hline Traumatology & $264(12)$ & $325(13)$ & $190(8.1)$ & $33(3)$ & $22(2.5)$ \\
\hline Urology & $97(4)$ & $91(3.7)$ & $91(4)$ & $50(4.5)$ & $40(4.6)$ \\
\hline Total & 2279 & 2486 & 2318 & 1102 & 861 \\
\hline \multicolumn{6}{|l|}{ Symptoms at triage, no. $(\%)^{*}$} \\
\hline Fever & $48(2.1)$ & $86(3.4)$ & $105(4.5)$ & $115(10.4)$ & $92(10.6)$ \\
\hline Cough & $60(2.6)$ & $89(3.6)$ & $78(3.3)$ & $107(9.7)$ & $18(2)$ \\
\hline Dyspnoea & $98(4.3)$ & $52(2)$ & $138(6)$ & $118(10.7)$ & $110(12.8)$ \\
\hline Chest pain & $66(2.9)$ & $67(2.7)$ & $67(2.9)$ & $18(1,6)$ & $12(1,4)$ \\
\hline \multicolumn{6}{|l|}{ Severity code at triage, no. $(\%)$} \\
\hline Red & $31(1.4)$ & $38(1.5)$ & $36(1.6)$ & $43(3.9)$ & $23(2.7)$ \\
\hline Yellow & $359(15.7)$ & $412(17)$ & $365(15.7)$ & $250(22.7)$ & $129(15)$ \\
\hline Green & $1245(54.6)$ & $1431(57.5)$ & $1174(50.7)$ & $666(60.4)$ & $542(63)$ \\
\hline White & $644(28.3)$ & $605(24)$ & $743(32)$ & $143(13)$ & $167(19.3)$ \\
\hline Men, no. $(\%)$ & $948(41.6)$ & $1019(41)$ & $987(42.6)$ & $513(46.5)$ & $328(38)$ \\
\hline Median age, years & 52 & 53 & 51 & 58 & 57 \\
\hline Death in ED, no. (\%) & $7(0.3)$ & $12(0.5)$ & $16(0.7)$ & $48(4.3 \%)$ & $28(3.2)$ \\
\hline Hospitalization rate, no. (\%) & $294(13)$ & $302(12.1)$ & $305(13.1)$ & $290(26.3)$ & $241(28)$ \\
\hline
\end{tabular}

*As the predominant presentation symptom 
All these data are justified by the increased number of patients with pneumonia and ARDS who were admitted to $\mathrm{ED}$, which also explains the increased hospitalization rate as well as the rate of patients who died in ED (from a mean of $13 \% /$ month to $26 \%$ and $28 \%$, and from a mean of $0.5 \% /$ month to $4.3 \%$ and 3.2\% in March and April 2020, respectively) due to the greater severity of disease such in patients.

Another aspect that may have had a negative impact on patient's health could be linked to a reduced drug intake. Anxiety about the period we are experiencing and the impossibility of getting regular medical care and prescriptions for adequate supplies may have caused a reduced adherence to treatment [6]. Furthermore, the monitoring of some life-saving drugs such as oral anticoagulants, immunosuppressants or anticomitial may have been reduced. For all these reasons, it is possible that some clinical conditions progressed until they became a real urgency. A recent study showed that the mortality from acute heart attack in Italy has almost tripled for late clinical presentation caused by patients avoiding hospitals for fear of SARS-CoV-2 infection [7], a phenomenon that is being observed also in other European countries [8] and in the USA [9].

To prevent this in the near future, patients with chronic conditions should be at least monitored and managed with telephone or online health consultation, identifying those who need urgent access to care. Prioritizing outpatient visits based on disease severity in patients with chronic diseases already in regular follow-up could be done through semi-automated artificial intelligence systems to avoid further worsening of the underlying diseases. Patients with mild conditions could on the other hand be managed outside the hospital by implementing telemedicine and creating networks of general practitioners who can consult with in-hospital specialists. Based on our own experience, we believe that to enable this, it is of key importance identifying dedicated medical personnel (physician and nurses) who can take care of these patients' selection. Telemedicine services eliminate the need to address patients to overpopulated hospitals and provide near real-time consultation. To do this, we must not rely only on individual willing, but plan with the health policymakers the best management strategies, also endowing this with a medico-legal value.

For all hospital-dispensing drugs, it is necessary to guarantee a timely supply, sending recipe codes or directly the drugs to the patient's home, avoiding unnecessary access to the hospital and, as much as possible, stressing the importance of compliance to treatment, which could decrease the generalized anxiety [6].

There is an urgent need to manage all patients with emergencies as well as the most fragile ones who need urgent access to care, with appropriate standards of care and dedicated preventive measures and protocols against the risk of SARS-CoV-2 infection. We must reduce as much as possible and as soon as possible the risk of acquiring in-hospital infection, especially considering that asymptomatic cases may be a vector of the virus and can infect medical and nursing staffs. The goal is to guarantee the best and safest treatment to all patients who will return to hospitals to be treated according to the recommendations and the published guidelines [10-12].

We believe that all patients hospitalized urgently should have a swab and a chest X-ray as soon as they enter the ED and have the result before admission to the ward, while those considered for elective procedures should have the swab performed the day before admission. In case of a negative swab and chest X-ray result and in the absence of suspicious symptoms of virus infection, the patients can be admitted to the ordinary ward, where only SARS-CoV-2-negative patients are hospitalized, whereas in case of a positive evaluation the patients should be placed in the ward dedicated only to SARS-CoV-2-positive patients, with separate staff of nurses and physician.

If we will be able to do all this in the next few weeks, we will respond to their medical needs, avoiding an excessive increase in morbidity and mortality.

Funding None.

\section{Compliance with ethical standards}

Conflict of interest M. Viganò: Board/speaker Bureau for BMS, MSD, Alfasigma, Gilead Scinces, Fujirebio and Intercept. S. Harari: Grants and personal fees from Roche, Actelion and boehringer Ingelheim.

Statement of human and animal rights This study was conducted in accordance with the ethical standard of our Institutional committeee on Human experimentation.

Informed consent None.

\section{References}

1. Guan WJ, Ni ZY, Hu Y et al (2020) Clinical characteristics of Coronavirus Disease 2019 in China. N Engl J Med 382:1708-1720

2. Grasselli G, Pesenti A, Cecconi M (2020) Critical care utilization for the COVID-19 outbreak in Lombardy, Italy: early experience and forecast during an emergency response. JAMA 323:1574-1581

3. Vitacca M, Nava S, Santus P, Harari S (2020) Early consensus management for non-ICU ARF SARS-CoV-2 emergency in Italy: from ward to trenches. Eur Respir J 55:2000632

4. Istituto Superiore di Sanità. "COVID-19 epidemic. 2 April 2020 national update" Appendix to the bulletin with a regional breakdown. https://www.epicentro.iss.it/coronavirus/bollettino/Bolle tino-sorveglianza-integrataCOVID-19. Accessed 25 Apr 2020

5. Viganò M, Voza A, Harari S et al (2020) Clinical management of non-respiratory diseases in the COVID-19 pandemic: what have 
we done and what needs to be done. Telemed J E Health. https:// doi.org/10.1089/tmj.2020.0148(Online ahead of print)

6. Brooks SK, Webster RK, Smith LE et al (2020) The psychological impact of quarantine and how to reduce it: rapid review of the evidence. Lancet 395(10227):912-920

7. Cosentino N, Assanelli E, Merlino L et al (2020) An in-hospital pathway for acute coronary syndrome patients during the COVID19 outbreak: initial experience under real-world suboptimal conditions. Can J Cardiol. https://doi.org/10.1016/j.cjca.2020.04.011

8. Rodríguez-Leora O, Cid-Álvarezd B, Ojedae S et al (2020) Impacto de la pandemia de COVID-19 sobre la actividad asistencial en cardiología intervencionista en España. REC Interv Cardiol. https://doi.org/10.24875/RECIC.M20000120.5

9. Garcia S, Albaghdadi MS, Meraj PM et al (2020) Reduction in ST-segment elevation cardiac catheterization laboratory activations in the United States during COVID-19 pandemic. J Am Coll Cardiol. https://doi.org/10.1016/j.jacc.2020.04.011

10. Friese CR, Veenema TG, Johnson JS, Jayaraman S, Chang JC, Clever LH (2020) Respiratory Protection Considerations for
Healthcare Workers During the COVID-19 Pandemic. Health Secur. https://doi.org/10.1089/hs.2020.0036[Epub ahead of print]

11. Verbeek JH, Rajamaki B, Ijaz S et al (2020) Personal protective equipment for preventing highly infectious diseases due to exposure to contaminated body fluids in healthcare staff. Cochrane Database Syst Rev. 4:CD011621. https://doi.org/10.1002/14651 858.CD011621.pub4

12. Dexter F, Parra MC, Brown JR, Loftus RW (2020) Perioperative COVID-19 Defense: an evidence-based approach for optimization of infection control and operating room management. Anesth Analg. https://doi.org/10.1213/ANE.0000000000004829 [Epub ahead of print]

Publisher's Note Springer Nature remains neutral with regard to jurisdictional claims in published maps and institutional affiliations. 